\title{
Multi-Band Transceiver for Tracking Application
}

\author{
Amit Naik \\ Communication Laboratory, Electronics Engineering \\ Defence Institute of Advanced Technology, \\ DRDO Lab \\ Girinagar, Pune 411025
}

\author{
K. Krishna Naik, PhD \\ Communication Laboratory, Electronics Engineering \\ Defence Institute of Advanced Technology, \\ DRDO Lab \\ Girinagar, Pune 411025
}

\begin{abstract}
Conventional Transceivers are designed using particular wireless technology and thus works in a specified range. This paper presents data transmission over Encrypted Multiband Transceivers which makes use of different wireless communication technologies, Encryption and GPS is used to securely communicate an image or other kinds of digital information with efficiency. It uses dynamic switching which is depending upon the distance, network, receiver sensitivity etc. Transceiver communication protocols are discussed with the help of software implementation and their algorithms.
\end{abstract}

\section{Keywords}

Bluetooth; Transceiver; WLAN; ZigBee

\section{INTRODUCTION}

Technologies provided in mobile such as Bluetooth, GSM, WLAN allow users to connect wirelessly [1]. Depending upon the prevailing environment such as the availability of the network, service or technology, distance, receiver sensitivity etc. In a wireless telecommunication network dual-band, tri-band, multi-band devices are used which are based on multi-band concept. These wireless devices work on multiple radio frequency bands and multiple channels operating mobile devices use multiple frequencies [2]. A suitable technology within the multiband transceiver will automatically switch to Bluetooth, GSM, WLAN or ZigBee. This will reduce the rush on the bandwidth and the cost of transferring data will be very low. The band consists of a group of frequencies which contains a number of channels. The multi-bands are extensively used to provide the parallel transmission, parallel reception of signal circuit path and frequency. This phenomenon leads to the increase in demand of power, efficiency, cost and the complexity in multi-band operated mobile devices.
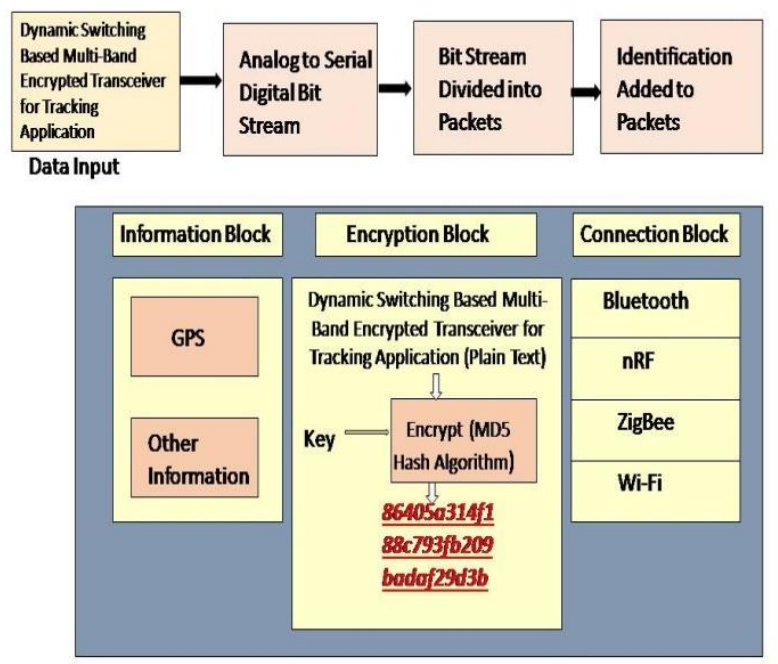

Fig. 1 Block diagram of data transmission over multi-band transceiver
There are different wireless technologies like Bluetooth, Wi-Fi, GSM and ZigBee which allow the proper wireless communication between two mobile phones. These types of wireless communication technology have different ranges to connect the two wireless devices like Bluetooth works on a 2.45 $\mathrm{GHz}$ frequency band. Wi-Fi operates on two frequency bands of $5 \mathrm{GHz}$ and $2.4 \mathrm{GHz}$. On the other hand, Wi-Max works in two frequency bands, $2-11 \mathrm{GHz}$ and $10-66 \mathrm{GHz}$ [1]. This paper presents transmission of text in wireless communication medium with dynamic switching; first input text is converted into a serial digital bit stream. Bit stream is divided into data packets. Identification is added in these packets and identification can be specification about packet like the sent location of the packet, sent device etc. This process can be performed with information block which consists of two parts; first one is GPS which stands for Global Positioning System. Modern GPS is capable of giving the accurate location with 1 meter tolerance. This application uses mobile's GPS for tracking user's real-time location [5]. It also stores some other useful information about users like packet size and storage capacity. Since this information is sensitive so it becomes necessary to secure the information. Then it is transmitted in encrypted form so that even if the information is hacked, there will be no compromise with privacy. Fig. 1 shows the block diagram of data transmission over multiband transceiver.

Encryption can be defined as a procedure of converting data or signal into an unreadable form using some algorithms for restricting unauthorized access. Encryption protects sensitive information such as Credit card number, bank password etc by encoding them and adding cipher text in it. This encrypted data can only be decrypted by an encryption key. Key and Cipher are two important part of an encryption operation [4]. In this paper, we are using Message Digest Algorithm 5 (MD5) as cryptographic hash function. MD5 is also used to generate a file of a fingerprint to confirm that a made file is same after the transfer of signal. In this paper MD5 hash consists of 32 hexadecimal characters. We can also transmit image, audio and video as an input. Digital images are a numeric symbol, usually binary, of a two-dimensional symbol or image. A system method for digital image transmission includes a transceiver for transfer and reception channel valuation signals and receiving a digital symbol or image file and a memory for loading the received digital image file.

In digital communication, we can transmit any number of digital files which can be transmitted or received over the computer networks. Day by day multimedia applications is increasing rapidly like video, audio and images etc. So security becomes an important aspect of digital communication. Many encryption methods are discovered to ensure the security features and here image encryption is one of them for security purpose. This evaluation technique is used to transform the given original image or signal to another image which is unable to recognized and hard to understand for users. 


\section{SOFTWARE IMPLEMENTATION AND WORKING}

With the idea of the concept of multiband transmission, wireless system unit consists of two transceivers. In Transceiver 1 , at one end the data to be transmitted is fed to the transmitting device and simultaneously to a frequency to voltage converter device, the voltage data is fed to microcontroller this helps to categorize the frequency band in which the data is to be transmitted, also this ADC data is sent separately to the receiver either by wired or any wireless medium which acts as a synchronization signal and helps to recognize the frequency band to be used at the receiver end. At the transmitter section, three wireless modules namely Bluetooth, XBee and Wi-Fi are connected and operated via a DE multiplexer which acts as a switch and connects the module to the UART of the microcontroller according to the $\mathrm{ADC}$ value and then starts transmitting the data [1].

In transceiver 2, at the receiver end, the same concept is applied and the DE multiplexer switch connects the module to the receiver microcontroller and starts receiving the data which can be displayed on any device. For making both the end as a transceiver, the roles played by both the ends are interchanged. The software implementation is performed in Proteus. After receiving the output, we performed decryption to get original form of input data. The modules and their frequency range are given below.

Table 1: Frequency ranges and other parameters of Modules [1] [6]

\begin{tabular}{|c|c|c|c|}
\hline Parameter & Bluetooth & Zigbee & Wifi \\
\hline Range & $0-10$ meters & $\begin{array}{l}\text { 10meter-100 } \\
\text { meters }\end{array}$ & $\begin{array}{l}\text { 50meter-100 } \\
\text { meters }\end{array}$ \\
\hline $\begin{array}{l}\text { Operating } \\
\text { frequency } \\
\text { Range } \\
\text { (Considered) } \\
\text { (Modules) }\end{array}$ & $\begin{array}{l}2402-2480 \\
\mathrm{MHz}\end{array}$ & $902-928 \mathrm{MHz}$ & $\begin{array}{l}2.4 \\
/ 3.6 / 4.9 / 5 / 5.9 \\
\mathrm{GHz}\end{array}$ \\
\hline Data type & $\begin{array}{l}\text { File, graphic, } \\
\text { picture, } \\
\text { audio }\end{array}$ & $\begin{array}{l}\text { Data in small } \\
\text { packets }\end{array}$ & $\begin{array}{l}\text { Audio, } \\
\text { graphics, file, } \\
\text { picture, video }\end{array}$ \\
\hline Complexity & $\begin{array}{l}\text { Very } \\
\text { complex }\end{array}$ & Simple & Very complex \\
\hline Applications & $\begin{array}{l}\text { replacement } \\
\text { of cabling }\end{array}$ & $\begin{array}{l}\text { automation, } \\
\text { control }\end{array}$ & $\begin{array}{l}\text { Web, e-mail, } \\
\text { video }\end{array}$ \\
\hline $\begin{array}{l}\text { Power } \\
\text { consumption }\end{array}$ & High & Low & High \\
\hline $\begin{array}{l}\text { Maximum } \\
\text { signal rate }\end{array}$ & $1 \mathrm{Mb}$ per sec & $250 \mathrm{~kb}$ per sec & $54 \mathrm{Mb}$ per sec \\
\hline $\begin{array}{l}\text { Nodes per } \\
\text { master }\end{array}$ & 7 & 64000 & 32 \\
\hline Battery life & 1 Week & $>1$ Year & Hours \\
\hline $\begin{array}{l}\text { Operating } \\
\text { temperature }\end{array}$ & $\begin{array}{l}32^{0} \mathrm{~F} \text { to } 122^{0} \\
\mathrm{~F}\end{array}$ & $\begin{array}{l}0 \text { to } 55^{0} \mathrm{C}\left(32^{0} \mathrm{~F}\right. \\
\left.\text { to } 131^{0} \mathrm{~F}\right)\end{array}$ & $32^{0} \mathrm{~F}$ to $104^{0} \mathrm{~F}$ \\
\hline $\begin{array}{l}\text { Bandwidth } \\
(\mathrm{kb} / \mathrm{s})\end{array}$ & 720 & $20-250$ & 11000 \\
\hline Topology & Star & Star, mesh, cluster & Star \\
\hline Advantages & $\begin{array}{l}\text { low cost, } \\
\text { easy to use }\end{array}$ & $\begin{array}{l}\text { low cost, reliable, } \\
\text { good performance }\end{array}$ & $\begin{array}{l}\text { data rate, } \\
\text { flexibility }\end{array}$ \\
\hline
\end{tabular}

\section{ALGORITHMS}

The algorithms and Programming logic for both transceivers are

Algorithm for Transceiver 1

Initial setup

\{Controller setup\}

\{Module setup\}

Initialize the controller and provide the data

Read ADC

If-else

\{

Control logic

Synch. data send

Module data send

\}

\}

Algorithm for Transceiver 2

Initial setup

\{Controller setup\}

\{module setup\}

Initialize the controller

Read synch-data

If-else

\{

Control logic

\{

Read Module data

\}

\}

[3] Programming Logic:

\section{Transmitter}

First of all we declared global variables and include standard Input/Output functions. Transmitter programming logic is:

1. a=read_adc(0);

2. $\mathrm{OCR} 1 \mathrm{~A}=\mathrm{a}$;

3. $\operatorname{if}(\mathrm{a}<80)\{$

4. PORTD. $2=0$;

5. ORTD. $3=0 ;\}$

6. else if $(a>=80 \& \& a<140)\{$

7. PORTD. $2=0$;

8. PORTD. $3=1 ;\}$

9. else if $(a>=140 \& \& a<250)\{$

10. PORTD.2=1;

11. PORTD. $3=0 ;\}$ 

12. else if $(a>=80 \& \& a<140)\{$
13. PORTD. $2=1$;
14. PORTD. $3=1 ;\}$
15. putchar(data);\}\}

\section{$\underline{\text { Receiver }}$}

Receiver will follow the same starting process like transmitter. First of all we declared global variables and include standard Input/ Output functions. Receiver programming logic is:
1. $\quad a=r e a d \_a d c(0)$;
2. $\quad$ if $(a<80)\{$
3. PORTD. $2=0$;
4. PORTD. $3=0 ;\}$
5. else if $(a>=80 \& \& a<140)\{$
6. PORTD. $2=0$;
7. PORTD. $3=1 ;\}$
8. else if $(a>=140 \& \& a<250)\{$
9. PORTD. $2=1$;
10. PORTD. $3=0 ;\}$
11. else if $(a>=80 \& \& a<140)\{$
12. PORTD. $2=1$;
13. PORTD. $3=1 ;\}$
14. $\operatorname{ch}=$ getchar();
15. lcd_putchar(ch);\}\}
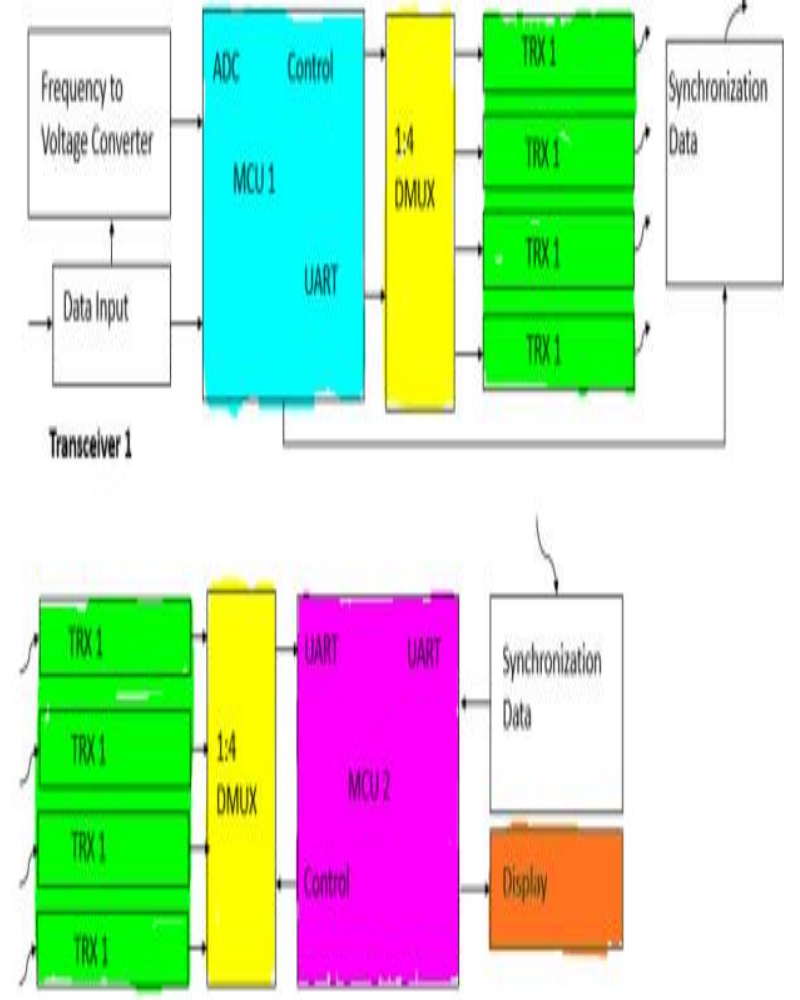

Inssaviver?

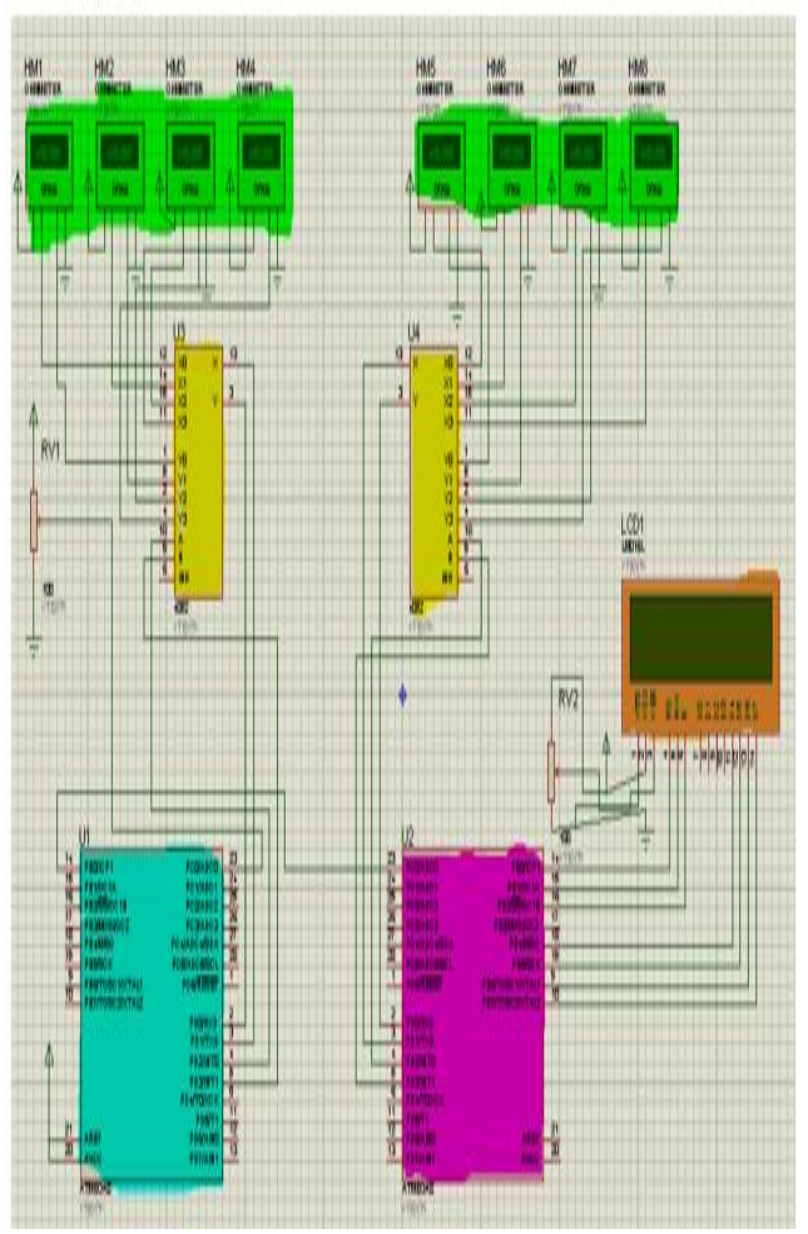

Fig.2: Mapping of Block Diagram with Software implementation $[2,3]$ 


\section{CONCLUSION}

An efficient and secure multiband transceiver is designed and illustrates the process of transmitting a text over the network. This application emphasizes on secured encrypted communication to ensure a high level of privacy. This application allows the user to connect according to their apart radius. The concept of software programming in a multiband transceiver is used for communication between two devices. This phenomenon provides the fast convergence, flexibility in wireless communication technology. The scanning of Receiver Sensitivity and Distance make the communication very easy between two mobile phones. By GPS and other information location, authenticity and tracking are possible. The use of multi-band transceiver provides the fast and great connectivity, minimum cost, and better adaptability. This procedure minimizes the high volume traffic in a wireless communication network.

\section{REFERENCES}

[1] J. Ryynanen, S. Lindfors, K. Stadius and K. A. I. Halonen, "Integrated circuits for multiband multimode receivers," in IEEE Circuits and Systems Magazine, vol. 6, no. 2, pp. 5-16, 2006.

[2] A.Naik and K.K. Naik "Handover in multiband Transceiver,"2016 International Conference on ICT in Business Industry and Government(ICTBIG),Indore 2016,pp1-4.dio:10.1109/ICTBIG.2016.7892654 .

[3] Bo Su and Li Wang, "Application of Proteus virtual system modelling (VSM) in teaching of microcontroller," 2010 International Conference on E-Health Networking Digital Ecosystems and Technologies (EDT), Shenzhen, 2010, pp. 375-378.

[4] Z. Yong-Xia and Z. Ge, "MD5 Research," 2010 Second International Conference on Multimedia and Information Technology, Kaifeng, 2010, pp. 271-273.doi: 10.1109/MMIT.2010.186

[5] K. Dhinakaran, S. Srinath, S. Sriram and R. Venkateshwar, "GPS based tracking system for transit objects," 2017 Third International Conference on Science Technology Engineering \& Management (ICONSTEM), Chennai, 2017, pp. 194-201.

[6] Annual report of ICT (Information and communication Technology) facts and figures 2017.

[7] Press Release No. 43/2017 of Telecom Regulatory
Authority of India (TRAI).

[8] M. Ismail, M. Kashef, E. Serpedin and K. Qaraqe, "On balancing energy efficiency for network operators and mobile users in dynamic planning," in IEEE Communications Magazine, vol. 53, no. 11, pp. 158-165, November 2015.

[9] M. Polese, M. Giordani, M. Mezzavilla, S. Rangan and M. Zorzi, "Improved Handover Through Dual Connectivity in 5G mmWave Mobile Networks," in IEEE Journal on Selected Areas in Communications, vol. 35, no. 9, pp. 2069-2084, Sept. 2017.

[10] M. Biagi, F. Cuomo, M. Perri and A. Irjoob, "A MultiLayer Parametric Approach to Maximize the Access Probability of Mobile Networks," in IEEE Access, vol. 4, pp. 6692-6703, 2016.

[11] Sagar Shriram Salwe, K. Krishna Naik, "Discrete Image Data Transmission in Heterogeneous Wireless Network using Vertical Handover Mechanism," IET Image Processing vol.11 no.7, pp.550-558, 2017. DOI: 10.1049/iet-ipr.2016.0779.

[12] Sagar Shriram Salwe, K. Krishna Naik, "Heterogeneous Wireless Networks for IoT Applications," IETE Technical Review pp.1-8, 2017.DOI:10.1080/02564602.2017.1400412.

[13] J. Lee, Y. Su and C. Shen, "A Comparative Study of Wireless Protocols: Bluetooth, UWB, ZigBee, and WiFi," IECON 2007 - 33rd Annual Conference of the IEEE Industrial Electronics Society,Taipei,2007,pp.4651.doi:10.1109/IECON.2007.4460126.

[14] Karunakar Pothuganti and Anusha Chitneni "A Comparative Study of wireless Protocols:Bluetooth ,UWB,ZigBee and Wi-Fi"Advance in Electronic and Electric Engineering,ISSN 2231-1297,volume 4,number 6(2014),pp 655-662.

[15] E. Ferro and F. Potorti, "Bluetooth and Wi-Fi wireless protocols: A survey and a comparison," IEEE Wireless Commun., vol. 12, no. 1, pp. 12-16, Feb. 2005.

[16] X. Wang, Y. Ren, J. Zhao, Z. Guo, and R. Yao, "Comparison of IEEE 802.11e and IEEE 802.15.3 MAC," in Proc. IEEE CAS Symp. Emerging Technologies: Mobile \& Wireless Commun, Shanghai, China, May, 2004, pp. 675-680. 